\title{
Factors associated with suggestive of pulmonary hypertension measured by echocardiography in patients with a mediastinal tumor: A single-center study
}

\author{
Dong Geum Shin ${ }^{1}$, Min-Kyung Kang ${ }^{2}$, Kun Il Kim ${ }^{1}$, Hodong Yang${ }^{1}$, Donghoon Han ${ }^{1}$, \\ Seonghoon $\mathrm{Choi}^{1}$, Jung-Rae $\mathrm{Cho}^{1}$, and Namho Lee ${ }^{1}$ \\ ${ }^{1}$ Affiliation not available \\ ${ }^{2}$ Kangnam Sacred Heart Hospital
}

January 20, 2021

\begin{abstract}
Background: Ultrasound techniques are generally not used as a primary tool in the evaluation of mediastinal tumors and cysts. This study aimed to identify factors associated with pulmonary hypertension (PH) measured by transthoracic echocardiography (TTE) in patients with a mediastinal tumor. Methods: This retrospective, observational study was performed from January 2015 to December 2020. Fifty-five patients (mean age, $62 \pm 13$ years; 31 [56\%] women) who had a mediastinal tumor and underwent TTE were included. Patients were classified as with PH or without PH. We analyzed clinical factors and echocardiographic parameters. Results: PH was found in $21(38 \%)$ patients. Twenty-two patients were asymptomatic, and none had symptoms associated with PH. Forty-seven (86\%) patients underwent surgery, and 23 (42\%) patients were diagnosed with malignant tumors. The presence of PH was not related with malignancy. Patients with $\mathrm{PH}$ were older than those without $\mathrm{PH}(67 \pm 10$ versus [vs.] $59 \pm 14$ years, $\mathrm{p}=0.017)$. Small left ventricular $(\mathrm{LV})$ systolic dimension $(29.4 \pm 3.6$ vs. $31.6 \pm 3.6 \mathrm{~mm}, \mathrm{p}=0.040)$ and dimension $(4.2 \pm 0.3$ vs. $4.5 \pm 0.3 \mathrm{~mm}, \mathrm{p}=0.004)$ and hyperdynamic $\mathrm{LV}$ ejection fraction $(\mathrm{EF}, 69 \pm 6 \mathrm{vs} .65 \pm 5 \%, \mathrm{p}=$ 0.019) were associated with PH. Among them, older age, small LV dimension, and high EF were independently associated with PH. Conclusion: The presence of PH had no significant effect on patients' clinical manifestation or malignancy.
\end{abstract}

\section{Hosted file}

MD.pdf available at https://authorea.com/users/390735/articles/504966-factors-associatedwith-suggestive-of-pulmonary-hypertension-measured-by-echocardiography-in-patients-witha-mediastinal-tumor-a-single-center-study 


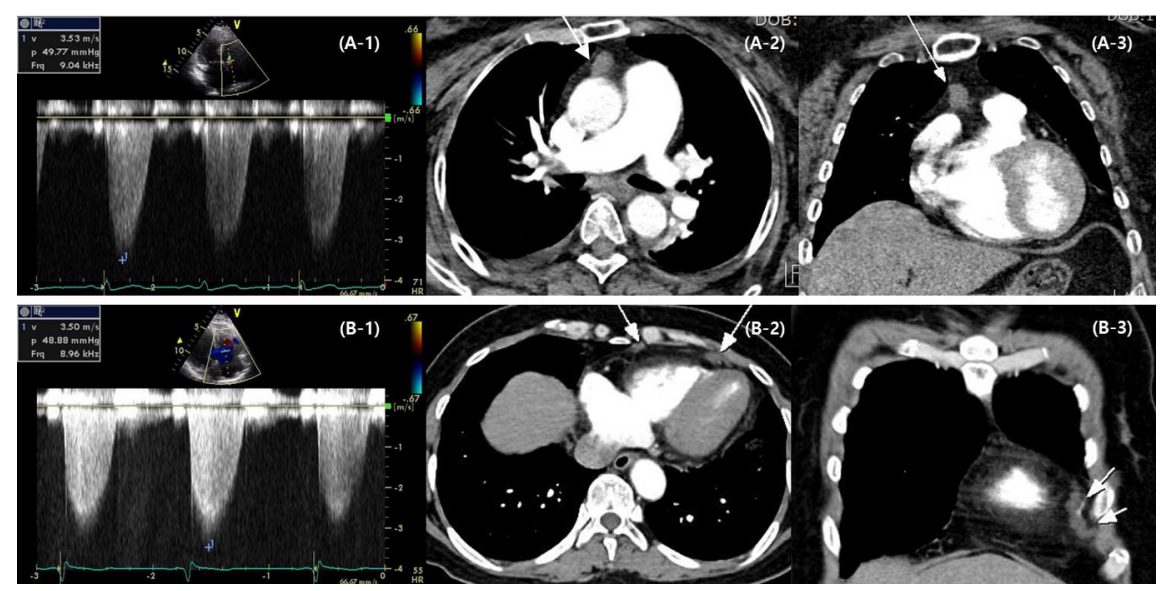

